\title{
Novel empirical correlations for estimation of bubble point pressure, saturated viscosity and gas solubility of crude oils
}

\author{
Ehsan Khamehchi', Fariborz Rashidi' ${ }^{*}$, Hanieh Rasouli' ${ }^{1}$ and Amir Ebrahi- \\ mian $^{2}$
}

${ }^{1}$ Faculty of Chemical Engineering, Amirkabir University of Technology, Tehran, Iran
${ }^{2}$ Faculty of Petroleum Engineering, Amirkabir University of Technology, Tehran, Iran

\begin{abstract}
Knowledge of petroleum fluid properties is crucial for the study of reservoirs and their development. Estimation of reserves in an oil reservoir or determination of its performance and economics requires a good knowledge of the fluid physical properties. Bubble point pressure, gas solubility and viscosity of oils are the most important parameters in use for petroleum and chemical engineers. In this study a simple-to-use, straight-forward mathematical model was correlated on a set of 94 crude oil data. Three correlations were achieved based on an exponential regression, which were different from conventional empirical correlations, and were evaluated against 12 laboratory data other than those used for the regression. It is concluded that the new exponential equation is of higher precision and accuracy than the conventional correlations and is a more convenient mathematical formulation.
\end{abstract}

Key words: Bubble point pressure, saturated viscosity, gas solubility, empirical equation, exponential multiple regression

\section{Introduction}

Determining the fluid properties by utilizing the PVT analysis is considered to be essential in the study of petroleum reservoirs, the design of production equipment and the estimation of the recovery efficiency of a reservoir. In the absence of these data for one reason or another, correlations are used to estimate the physical properties of a petroleum fluid.

It is worth mentioning that during the study of pressure losses that occur in a multiphase flow situation, there is the need to know the physical properties of a reservoir fluid at different temperatures, which are always below the temperature of the reservoir.

These properties are also needed for the design of surface operation equipment or the study of reservoir flow performance. Therefore correlations for estimating the values of these properties must be used.

\section{Petroleum fluid properties}

Reservoir engineers need to predict PVT properties of petroleum fluids at given conditions of temperature and pressure. Though this can be determined through PVT analysis of fluid sample tests or can be calculated by using equation-

*Corresponding author. email: rashidi@aut.ac.ir

Received September 15, 2008 of-state based on computer codes if the fluid composition is known, this information is often unavailable particularly at the early stage of field development or needs to be verified, supported and supplemented during the course of field development. It is the task of empirical correlations then to estimate the petroleum fluid properties as a function of the reservoir's readily available characteristics (Ahmed, 1990).

\section{Methods for predicting bubble point pres- sure}

In a given hydrocarbon system the highest pressure at which the first gas bubble forms is called the bubble point or the 'saturation' pressure of that system. This can be experimentally obtained by constant composition expansion tests.

Standing (1981) proposed the following correlation:

$$
\begin{aligned}
& P_{b}=18.2\left[\left(R_{s} / \gamma_{g}\right)^{0.83}(10)^{a}-1.4\right] \\
& a=0.00091\left(T_{(\mathrm{R})}-460\right)-0.0125(\mathrm{API})
\end{aligned}
$$

Glaso (1981) also proposed the following correlation:

$$
\log \left(P_{b}\right)=1.7669+1.7447 \log \left(P_{b}^{*}\right)-0.30218\left[\log \left(P_{b}^{*}\right)\right]^{2}
$$

where $P_{b}^{*}$ is given by the following equation: 


$$
\begin{aligned}
& P_{b}^{*}=\left(\frac{R_{s}}{\gamma_{g}}\right)^{a}(T)^{b}(A P I)^{c} \\
& a=0.816, b=0.172, c=-0.989
\end{aligned}
$$

Furthermore, Marhoun (1988) proposed the following relationship based on Middle Eastern crude oils:

$$
\begin{aligned}
& P_{b}=a R_{s}^{b} \gamma_{g}^{c} \gamma_{o}^{d} T^{e} \\
& a=5.38088 \mathrm{E}-3, b=0.715082, c=-1.87784 \\
& d=3.1437, e=1.32657
\end{aligned}
$$

\section{Methods for predicting oil viscosity}

Viscosity of reservoir fluids is one of the factors that determine the degree of the fluids being mobilized within reservoir rock. Accurate prediction of viscosity should be made at a given temperature and pressure so that proper enhanced or natural oil recovery scenarios could be planned and right mechanisms could be utilized after satisfying predictions are proved through simulation.

Regardless of the specific lab experiments that are usually requested for a reservoir, one can find well-known correlations for this purpose in the literature.

For dead oil, Beal (1946) proposed a graphical correlation in 1946 that was later transformed into a mathematical form by Standing in 1981 (see Eq. 5):

$$
\mu_{o d}=\left(0.32+\frac{1.8 \times 10^{7}}{A P I^{0.453}}\right)\left(\frac{360}{T-260}\right)^{a}
$$

In which $a=10^{(0.43+0.83 / A P I)}$

Beggs and Robinson (1975) also proposed the following correlation (Eq. 6) for dead oil, while later Sutton and Farshad (1984) reported $114.7 \%$ of error for this correlation when tested against 93 crude samples from the literature.

$$
\begin{aligned}
& \mu_{\text {od }}=10^{x}-1 \\
& X=Y(T-460)^{-1.163} \\
& Y=10^{z} \\
& Z=3.0324-0.02023 A P I
\end{aligned}
$$

The correlations proposed for calculation of viscosity of saturated oils are commonly based on the viscosity of oil in dead state. Chew and Connally (1959) presented a correlation that was transformed into a mathematical equation later by Standing in 1981 (Eq. 7).

$$
\begin{aligned}
& \mu_{o b}=10^{a}\left(\mu_{o d}\right)^{b} \\
& a=R_{s}\left[2.2\left(10^{-7}\right) R_{s}-7.4\left(10^{-4}\right)\right] \\
& b=\frac{0.68}{10^{c}}+\frac{0.25}{10^{d}}+\frac{0.062}{10^{e}} \\
& c=8.62\left(10^{-5}\right) R_{s} \\
& d=1.1\left(10^{-3}\right) R_{s} \\
& e=3.74\left(10^{-3}\right) R_{s}
\end{aligned}
$$

Beggs and Robinson (1975) suggested the following correlation

$$
\begin{aligned}
& \mu_{o b}=a\left(\mu_{o d}\right)^{b} \\
& a=10.715\left(R_{s}+100\right)^{-0.515} \\
& b=5.44\left(R_{s}+150\right)^{-0.338}
\end{aligned}
$$

\section{Methods for predicting gas solubility}

Gas solubility $\left(R_{\mathrm{s}}\right)$ is a measure of the capability of oil to dissolve gas. It is in nature the result of complicated molecular interaction between all the individual components existing in oil and in associated gas. But, it is usually experimentally determined in the laboratory. For this oil property also there are empirical correlations presented in the literature.

Standing (1981) suggested the following equation:

$$
\begin{aligned}
& R_{s}=\gamma_{g}\left(\left(\frac{P}{18.2}+1.4\right) 10^{x}\right)^{1.2048} \\
& x=0.0125 \mathrm{API}-0.00091(T-460)
\end{aligned}
$$

Petrosky and Farshad (1993) suggested the following equation based on crude oil data from the Gulf of Mexico:

$$
\begin{aligned}
& R_{s}=\left[\left(\frac{P}{112.727}+12.340\right) \gamma^{0.8439} 10^{x}\right]^{1.73184} \\
& x=7.916\left(10^{-4}\right) A P I^{1.5410}-4.561\left(10^{-5}\right)(T-460)^{1.3911}
\end{aligned}
$$

\section{Multiple regression analysis}

Multiple regression analysis attempts to explain the relationships between the independent variables and a dependent variable. When there are $p$ independent variables $X_{1}, X_{2}, \cdots, X_{p}$, the multiple exponential regression equation is in the general form as follows:

$$
Y_{i}=\beta_{0} X_{1}^{\beta 1} X_{2}^{\beta 2} \cdots X_{p}^{\beta p}
$$

where, $Y$ is a dependent variable; $X_{1}, X_{2}, \cdots, X_{p}$ are independent variables; $\beta_{0}$ is the constant that the regression line intercepts the $\mathrm{Y}$ axis, representing the amount the dependent $Y$ will be when all the explanatory variables are 0 and $\beta_{i}, 1 \leq$ $i \leq p=$ the regression coefficient, representing the amount the response variable $Y$ changes when the explanatory variable changes 1 unit (Maason et al, 2003).

Regression estimation of the coefficients of an exponential model requires the use of logarithmic transformation applied to both sides of the equation. The results are:

$$
\begin{aligned}
& \log \left(Y_{i}\right)=\log \left(\beta_{0} X_{1}^{\beta 1} X_{2}^{\beta 2} \cdots X_{p}^{\beta p}\right) \Rightarrow \\
& \log \left(Y_{i}\right)=\log \left(\beta_{0}\right)+\beta_{1} \log \left(X_{1}\right)+\beta_{2} \log \left(X_{2}\right)+\cdots+\beta_{p} \log \left(X_{p}\right)
\end{aligned}
$$

The exponential function is linear in the logarithms of the dependent and independent variables. The coefficients $\beta_{1}$ and $\beta_{2} \cdots \beta_{p}$ are estimated directly in transformed regression. However, the transformed model constant, $\beta_{0}$, is the logarithm of the original equation constant. Thus the 
antilogarithm must be computed (Carlson and Thorne, 1997).

Table 1 summarizes the range of experimental data used for construction of the objective new correlations.

Table 1 The range of experimental data

\begin{tabular}{ccc}
\hline Property & Min & Max \\
\hline Gas solubility (SCF/STB) & 83 & 1708 \\
Gas gravity & 0.624 & 1.872 \\
Oil gravity & 0.554 & 0.858 \\
API gravity & 33.4 & 124 \\
Saturated oil viscosity, CP & 0.04 & 3 \\
Reservoir temperature, ${ }^{\circ} \mathrm{F}$ & 100 & 306 \\
\hline
\end{tabular}

\section{The new improved correlations (AUT Models)}

In this study, all the independent variables were selected for the three dependent output variables, namely bubble point pressure $\left(P_{\mathrm{b}}\right)$, oil viscosity $\left(\mu_{\mathrm{ob}}\right)$ and gas solubility $\left(R_{\mathrm{s}}\right)$. The logarithms Eq. 10 of all the variables were selected as inputs for analysis. Subsequently, a stepwise multiple linear regression analysis was carried out to determine the relationships between the dependent variable and the independent variables. To achieve this goal, regression analysis was carried out using the statistical software package SPSS for Windows. Stepwise regression procedures select the most correlated independent variable first, and then select the second independent variable which most correlates with the remaining variance in the dependent variable. This procedure continues until the selection of an additional independent variable does not increase the R-squared by a significant amount, usually a significance of at least $95 \%$.

The most reliable and meaningful regression equations that could be obtained by the statistical analysis are given as equations 11, 12 and 13:

$$
\begin{aligned}
& P_{b}=107.93 R_{s}^{0.9129} \gamma_{g}^{-0.666} T^{0.2122} A P I^{-1.08} \\
& \mu_{o b}=6.28 \times 10^{6} R_{s}^{-0.0266} T^{-1.032} A P I^{-2.786} \\
& R_{s}=0.0103 P_{b}^{1.014} \gamma_{g}^{0.719} T^{-0.223} A P I^{1.182}
\end{aligned}
$$

\section{Comparison between the AUT empirical equations and conventional models}

The statistical significance and validity of the presently derived model was checked by using some test statistics. The R-squared, also called the coefficient of determination, is the proportion of variance in the dependent variable, which can be predicted from the independent variables. The $R_{2}$ value calculated for the full models were $R_{2}=0.93,0.98$ and 0.95 for $P_{\mathrm{b}}, \mu_{\mathrm{ob}}$ and $R_{\mathrm{s}}$ respectively. This indicates that $93 \%$ of the variance of bubble point pressure, for example, can be predicted from variables $T, R_{\mathrm{s}}, \gamma_{\mathrm{g}}$ and $\gamma_{\mathrm{o}} . R$ is the square root of $R$-squared and is the correlation between the observed and predicted values of dependent variable. The calculated correlation coefficient $(R=0.93)$ indicates a strong relationship between the dependent and independent variables. The standard error of the estimate, also called the root mean square error, is the standard deviation of the error term, and is the square root of the mean square residual. The standard error of the estimate shows the standard deviation of the residuals to be $0.0610,0.0548$ and 0.0643 for $P_{\mathrm{b}}, \mu_{\mathrm{ob}}$ and $R_{\mathrm{s}}$ respectively.

For comparison purposes we used the models presented in sections 3 to 5 . The results of different models including the new AUT model (present study) are given in Figs. 1, 3 and 5. These figures are each sorted versus the experimental corresponding property and show how the new equations follow the real data in comparison with other correlations in use, for bubble point pressure, saturated oil viscosity and gas solubility respectively.

The derived equations were then exposed to some 12 evaluation data. This was done by applying about $10 \%$ of the available data for evaluation and thereby the equations were qualified. We used 12 randomly selected sets of experimental data among the 106 sets of data that were available. These test data are also well scattered in the whole range of avail-

\begin{tabular}{|c|c|c|c|c|c|c|}
\hline & $P_{\mathrm{b}}$ & Temp & $R_{\mathrm{s}}$ & Gas S. G & Oil S. G & API \\
\hline 1 & 400 & 219 & 123 & 0.835 & 0.8104 & 43.1 \\
\hline 2 & 550 & 211 & 145 & 0.897 & 0.8139 & 42.4 \\
\hline 3 & 650 & 290 & 251 & 0.755 & 0.6524 & 85.4 \\
\hline 4 & 795 & 219 & 248 & 0.887 & 0.7903 & 47.5 \\
\hline 5 & 855 & 200 & 223 & 0.722 & 0.7742 & 51.3 \\
\hline 6 & 985 & 200 & 264 & 0.712 & 0.7733 & 51.5 \\
\hline 7 & 1300 & 203 & 426 & 0.786 & 0.7592 & 54.9 \\
\hline 8 & 1450 & 237 & 699 & 0.827 & 0.6584 & 83.4 \\
\hline 9 & 1900 & 172 & 414 & 0.68 & 0.8134 & 42.5 \\
\hline 10 & 2415 & 220 & 464 & 0.836 & 0.847 & 35.6 \\
\hline 11 & 3000 & 185 & 811 & 0.812 & 0.842 & 36.6 \\
\hline 12 & 4200 & 200 & 891 & 0.975 & 0.887 & 28.0 \\
\hline
\end{tabular}
able data. The data used for the evaluation are given in Table 2 .

Table 2 Data used for the evaluation 
Figs. 2, 4 and 6 indicate that the proposed model could accurately predict $P_{\mathrm{b}}, \mu_{\mathrm{ob}}$, and $R_{\mathrm{s}}$ respectively. These scatter plots also show a comparison with other aforementioned correlations.

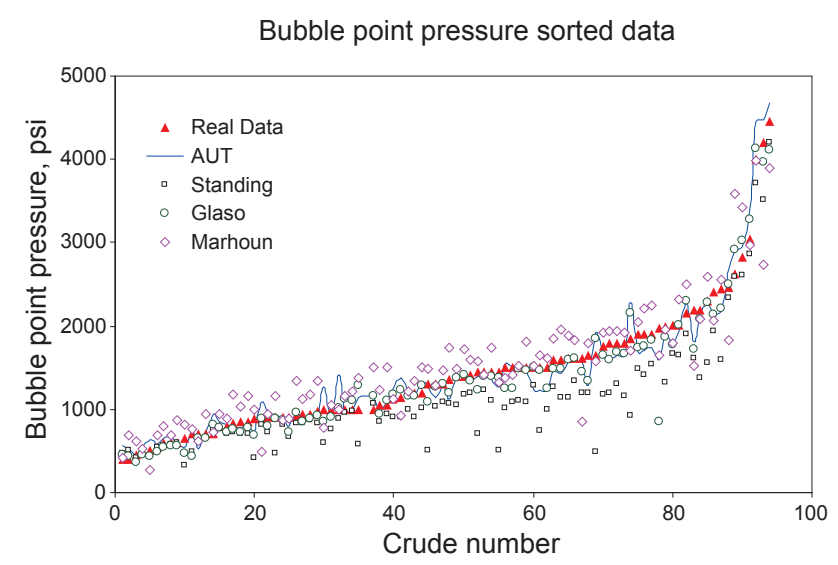

Fig. 1 Bubble point pressure vs. crude sample number

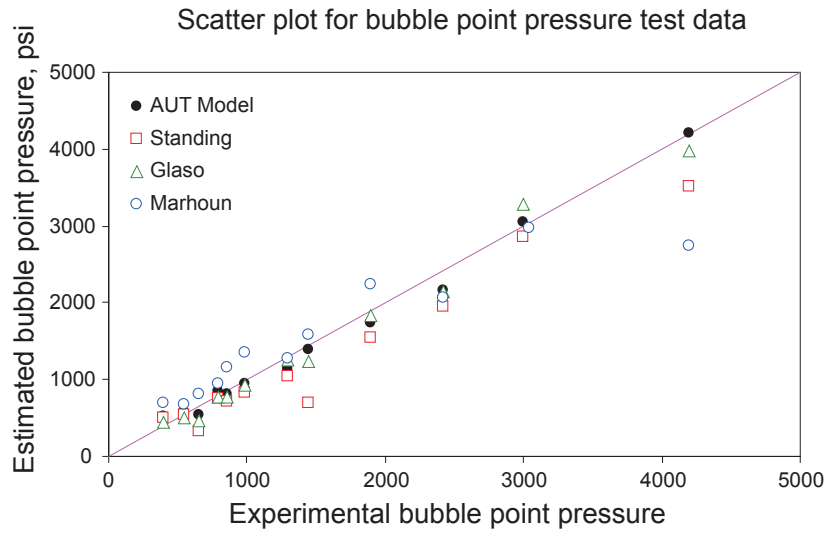

Fig. 2 Estimated vs. experimental $P_{\mathrm{b}}$ results for the testing data

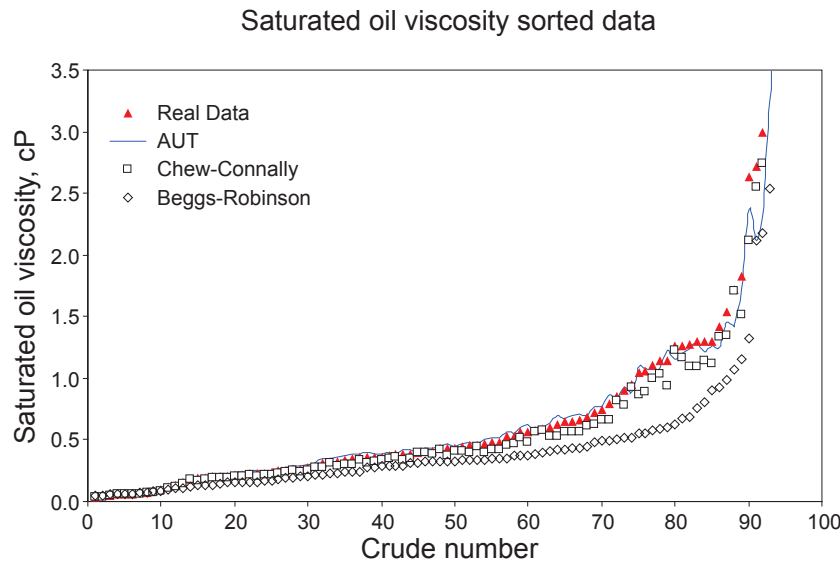

Fig. 3 Saturated oil viscosity vs. crude sample number
Scatter plot for satureted oil viscosity test data

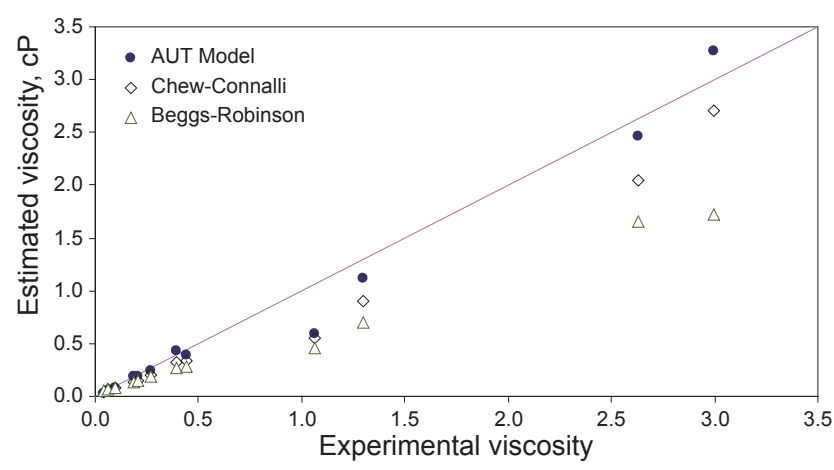

Fig. 4 Estimated vs. experimental $\mu_{\mathrm{ob}}$ results for the testing data

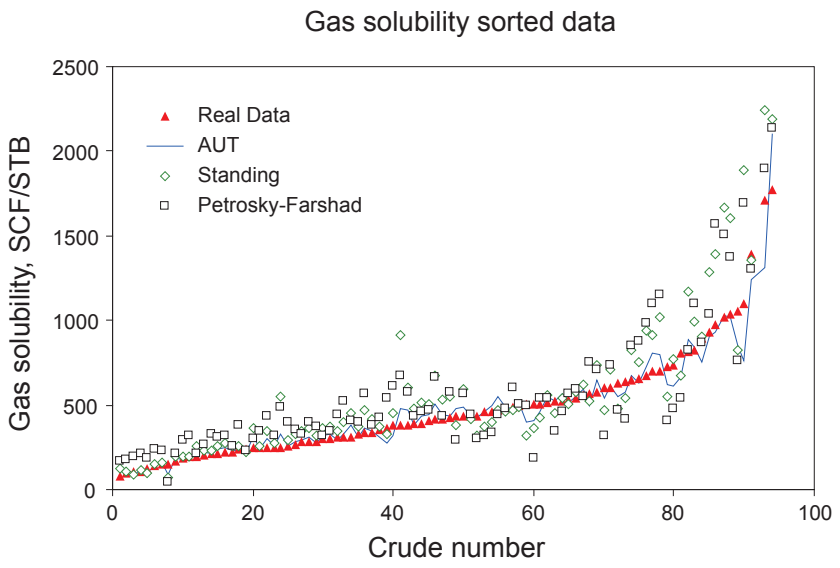

Fig. 5 Gas solubility vs. crude sample number

Scatter plot for gas solubility test data

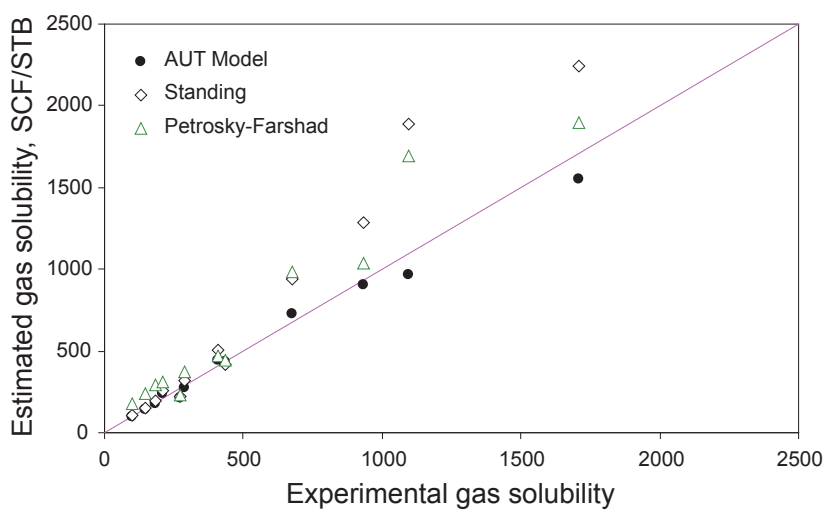

Fig. 6 Estimated vs. experimental $R_{\mathrm{s}}$ results for the testing data

\section{Conclusions}

In this study three simple to use, straight-forward empirical equations were proposed for estimation of bubble point pressure, saturated viscosity and gas solubility of crude oils respectively. The experimental data were correlated very well with the proposed correlations and the $\mathrm{R}^{2}$ index results were 
well near unity for all the bubble point pressure, saturated oil viscosity and gas solubility properties. While the reference data were extended over a wide span of magnitudes, the new models proved to be also of higher accuracy. This makes them more appropriate for applications compared to the conventional correlations.

\section{Nomenclatures}

API: American Petroleum Institute Index for oil gravity

$P$ : Pressure, psia

$P_{\mathrm{b}}$ : Bubble point pressure, psia

$R_{\mathrm{s}}$ : Gas solubility or gas oil ratio [Standard cubic foot per standard condition barrel], SCF/STB

SC: Ambient standard conditions in terms of temperature and pressure

$T$ : Temperature, ${ }^{\circ} \mathrm{F}$

$V_{\mathrm{o}}$ : Oil volume

$w$ : Connection weight

\section{Greeks}

$\gamma_{o}$ : Oil gravity

$\gamma_{g}$ : Gas gravity

$\mu_{\mathrm{od}}$ : Dead oil viscosity

$\mu_{\text {od }}$ : Saturated oil viscosity

\section{Subscripts}

b: Bubble Point

g: Gas

o: Oil s: Solubility

\section{Reference}

Ahmed T. Hydrocarbon Phase Behavior. Gulf Publishing. 1990. 1st Edition

Beal C. The viscosity of air, water, natural gas, crude oils and its associated gases at oil field temperatures and pressures. trans. AIME. 1946. 165. 94-112

Beggs H D and Robinson J R. Estimating the viscosity of crude oil systems. JPT. September 1975. 1140-1141

Carlson L W and Thorne B. Applied Statistical Methods for Business, Economics, and the Social Sciences. Prentice-Hall, Inc. 1997. 736-740

Chew J and Connally C A. A viscosity correlation for gas-saturated crude oils. Trans. AIME. 1959. 216. 23-25

Glaso O. Generalized pressure-volume-temperature correlations. JPT. May 1981. 785-795

Maason R L and Gunst R F and Hess J L. Statistical Design and Analysis of Experiments. John Wiley \& Sons Inc. 2003

Marhoun M A. PVT correlation for Middle East crude oils. JPT. May 1988. 650-665

Petrosky G E and Farshad F. Pressure-volume-temperature correlations for Gulf of Mexico crude oils. SPE Paper 26644.1993

Standing M B. Volumetric and Phase Behavior of Oil Field Hydrocarbon Systems. Society of Petroleum Engineers. 1981. 125-126

Sutton R P and Farshad F. Evaluation of empirically derived PVT properties for Gulf of Mexico crude oils. SPE Paper 13172. 1984

(Edited by Zhu Xiuqin) 